\title{
The Design of Multi-task Simulation Manipulator Based on Motor Imagery EEG
}

\author{
Yuhang $\mathrm{Ye}^{\S}$, Chenguang Yang ${ }^{\S \dagger *}$, Xinyang $\mathrm{Li}^{\ddagger}$, Zhaojie Jull, Zhijun $\mathrm{Li}^{\S}$

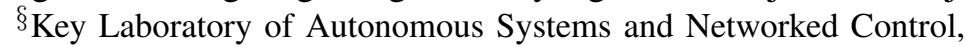 \\ College of Automation Science and Engineering, South China University of Technology, Guangzhou, 510640, China \\ ${ }^{\dagger}$ Zienkiewicz Centre for Computational Engineering, Swansea University, SA1 8EN, UK

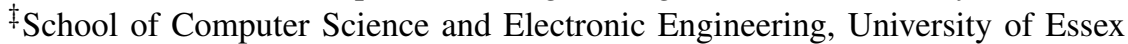 \\ $\|$ School of Computing, University of Portsmouth, Portsmouth PO1 3HE, U.K
}

\begin{abstract}
In this paper, a mind controlled multi-task manipulator based on motor imagery electroencephalogram (EEG) is proposed. Describe the system function first: In the case of only two types of control signal, the implementation of multi-task Manipulator relies on a toggle-confirmation mode of operation: the task is switched when imagining the left-hand movement, and the task is confirmed when the right-hand movement is imagined. In the BCI system, common spatial pattern (CSP) is used for feature extraction, mutual information for feature selection, and linear discriminant analysis (LDA) for pattern classification. The EEG signal is processed and classified into two categories, imagery of left-hand and right-hand movement. In this way, we can achieve the multi-task control of the manipulator under the premise of ensuring the accuracy of EEG recognition.
\end{abstract}

\section{INTRODUCTION}

Brain computer interface (BCI) is technology that enables their users to communicate and control external robotic systems using mental activity. [1] . It is an important means for people to understand and improve the function of the brain. Recently, technology of BCI developed rapidly. It had a wide range of applications in the field of control [2], rehabilitation of the disabled [3], entertainment [4], brain cognition and so on. At present, the application of EEG is mainly focused on two types of signals: evoked potential(EP) and spontaneous signal modulation. Evoked potential, including visual evoked potential [5], P300 event-related potential [6], is the electrical activity of the nervous system, which is stimulated by internal and external stimuli. But, spontaneous signal modulation, including the event related synchronization(ERS) and event related desynchronization(ERD) [7], spontaneous $\mu$ and $\beta$ rhythms [8], as well as slow cortical potential(SCP) [9], do not need any stimulation, simply by imagination, such as motor imagery.

In China, there are numerous patients with functional disability due to diseases or disorders such as spinal cord injury or amyotrophic lateral sclerosis (ALS). In recent years, there has been a lot of devices based on EEG that are designed for these

This work was partially supported by National Nature Science Foundation (NSFC) under Grant 61473120, Guangdong Provincial Natural Science Foundation 2014A030313266 and International Science and Technology Collaboration Grant 2015A050502017, Science and Technology Planning Project of Guangzhou 201607010006, and State Key Laboratory of Robotics and System (HIT) Grant SKLRS-2017-KF-13.

*Corresponding author. Email: cyang@ieee.org. people, such as mind controlled wheelchairs [10], artificial limbs [11], and manipulator [12]. However, non-invasive EEG devices collect the electrical signals generated by tens of thousands of neurons in the cerebral cortex [13], which have prominent non-linearity and non-stationary characteristics, so the brain-computer interface technology universally has two major problems: few recognition types and low recognition accuracy. These limitations make it difficult for EEG wheelchair, prostheses, and EEG robots to perform a variety of complex tasks. Therefore, these devices are difficult to be promoted and used for people with disabilities or in patients with motor disorders.

In this paper, we design a multi-task manipulator based on motor imagery because the EEG signal is only generated by imagination, does not depend on any sensory stimulation, which belongs to the true BCI system. In order not to affect the accuracy of EEG recognition, we use EEG algorithm of two-category classification, EEG signal after preprocessing, feature extraction, selection, and classification are divided into two categories: imaging left-hand movement or right-hand movement. In order to achieve multitask, we adopt a toggleconfirmation mode of operation: the task is switched when imagining the left-hand movement, and the task is confirmed when imagining the right-hand movement. For example, there are three tasks the disabled can select, including Taking medicine, drinking and first aid. If he/she want to drink, he/she images left-hand movement to switch the task to the "drinking", and then confirms this task by imaging righthand movement, after that the manipulator will take the cup to him/her. In this way, manipulator in accordance with the established mode of operation seem to lack the freedom of control, but it can solve a lot of problems when the manipulator is controlled directly by the EEG, such as difficult to control, lack of accuracy, error prone, etc.

The structure of this mind controlled Manipulator system is illustrated in Fig. 1. Neuroscan device is used for EEG signal collect with its attached software SCAN4.5. The EEG processing algorithm is implemented with Python for its concision to use, high efficiency of code execution and rich libraries especially the machine learning libraries. As a Communicator, MATLAB is responsible for the communication between the SCAN4.5, Python, and the simulation manipulator. So, the 


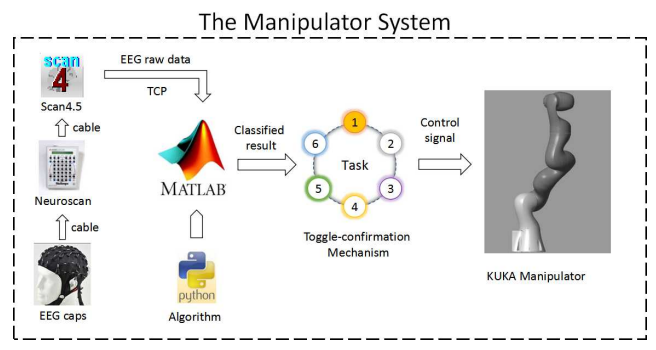

Fig. 1. System Framework

whole process is that: EEG signals are collected by Neuroscan and sent to SCAN4.5 by a cable. MATLAB communicates with SCAN4.5 by TCP client to get EEG data in real time. And then MATLAB calls Python program to process the raw EEG data and classify them into two categories. According to classified results, the multitasking simulation manipulator will run with the toggle-confirmation mechanism.

\section{The Motor IMAgery BCI}

\section{A. ERS/ERD Phenomenon in Motor Imagery}

When a certain area of cerebral cortex is activated, metabolism and information processing in this region will increase, leading to the amplitude reduction or block of the brain waves, especially in the alpha and beta rhythm. This electrophysiological phenomenon is called event related desynchronization (ERD) .On the contrary, when this region is at rest, the brain wave will show an obvious increase in amplitude, which is called the event related synchronization(ERS). Studies have shown that unilateral limb movement or motor imagery, the contralateral side of the brain produces ERD while the ipsilateral side of the brain produces ERS. It means that if we image right hand movement, the power of the EEG signals will reduce on the left side of the brain but increase on the right side of the brain, and vice versa. According to the ERS/ERD phenomenon, we can classify EEG signal into two categories, imaging left and right hand movement

\section{B. Better Data Preprocess with Optimal Feature Selection}

ERS/ERD phenomenons are usually more obvious in alpha $(8-12 \mathrm{~Hz})$ and beta $(18-26 \mathrm{~Hz})$ rhythm. But for different subjects, the most effective frequency bands are different. In order to optimize classification results of EEG data, it is important to obtain effective frequency bands for data processing. The method used for effective band selection in this paper are shown the Fig. 2. Firstly, there are 9 bandpass filters with $4 \mathrm{~Hz}$ bandwidth in turn from $4 \mathrm{~Hz}$ to $40 \mathrm{~Hz}$ for data preprocessing. Secondly, the filtered data will be put into feature extraction model. Finally, a method of mutual information can be used to select some feature which has a better distinction in ERS/ERD.

\section{Common Spatial Pattern Analysis for Feature Extraction}

In CSP, the objective is to find a desired spatial filters which are constructed as projection matrices [14]. Through the projection, the prominent ERD/ERS can be extracted by

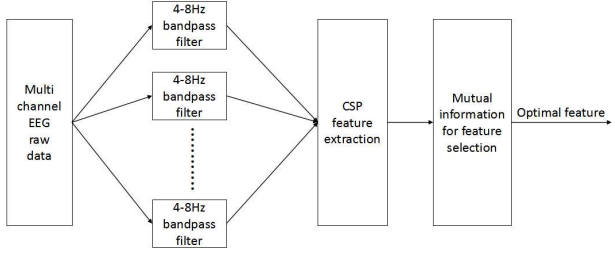

Fig. 2. Frequency band selection

maximizing the variance of one kind of EEG signal and minimizing the other one so that these two signals can be distinguished in the projected space.

Suppose that the one-dimensional filter is $\mathbf{w} \in \mathbb{R}^{n_{c} \times 1}$, and then the object function of CSP could be expressed as:

$$
\begin{array}{rll}
\mathbf{w}= & \arg \max \mathbf{w}^{T} \mathbf{E}\left[R \mid c_{1}\right] \mathbf{w} \\
\text { s.t. } & \mathbf{w}^{T}\left(\mathbf{E}\left[R \mid c_{1}\right]+\mathbf{E}\left[R \mid c_{2}\right]\right) \mathbf{w}=1
\end{array}
$$

where $\mathbf{E}\left[R \mid c_{i}\right]$ is the covariance matrix of EEG signals for class $c_{i}$, and $i=1,2$ representing different motor imagery classes. (1) could also be expressed in the following Rayleigh quotient forms:

$$
\mathbf{w}=\arg \max \frac{\mathbf{w}^{T} \mathbf{E}\left[R \mid c_{1}\right] \mathbf{w}}{\mathbf{w}^{T} \mathbf{E}\left[R \mid c_{2}\right] \mathbf{w}}
$$

Singular value decomposition could be used to obtain the spatial filters $\mathbf{w}$

$$
\mathbf{E}\left[R \mid c_{1}\right] \mathbf{w}=\lambda_{s} \mathbf{E}\left[R \mid c_{2}\right] \mathbf{w}
$$

where $\lambda_{s}$ is the eigenvalue of $\mathbf{E}\left[R \mid c_{1}\right]^{-1} \mathbf{E}\left[R \mid c_{2}\right]$, and they are sorted in a descending order, $s=1,2,3, \cdots, \xi$. According to how many feature to be extracted, $l$ pairs of $\mathbf{w}$ corresponding to $\left\{\lambda_{s}, s=1, \cdots, l, \xi-l+1, \cdots, \xi\right\}$ would be selected to form the projection matrices $W \in \mathbb{R}^{n_{c} \times 2 r}$. Thus, the features could be extracted as:

$$
\mathbf{f}=\operatorname{diag}\left(W^{T} R W\right)
$$

\section{Linear Discriminant Analysis for Classification}

Linear discriminant analysis (LDA) is a conventional pattern recognition algorithm. The main idea of LDA is to project the high-dimensional pattern samples into the optimal discriminant vector space. After projection, the samples in the new space have maximum inter-class distance and minimum intra-class distance, meaning that the pattern has the best separability in the new space. The object function of LDA could be expressed as:

$$
v=\arg \max \frac{v^{T} S_{b} v}{w^{T} S_{w} v}
$$

where $v$ is the projection matrices of LDA, $S_{b}$ and $S_{w}$ are the within-class scatter and between-class scatter of the two features extracted from the two type of EEG signals. (5) could be solved by solving a generalized eigenvalue problem

$$
S_{b} v=\lambda S_{w} v
$$




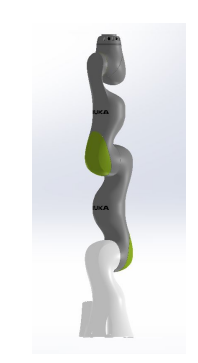

Fig. 3. The KUKA manipulator

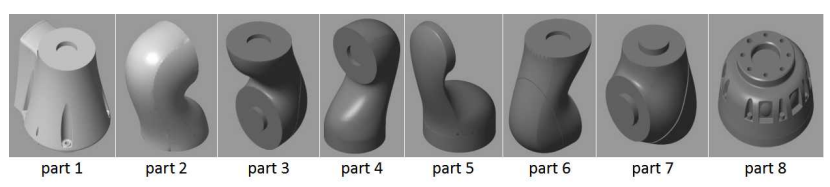

Fig. 4. Arm limb models of the KUKA manipulator

After that, a classification criterion is constructed, and the new feature from the newly collected EEG signal could be classified according to this criterion. This criterion can be expressed as:

$$
y(\mathbf{f})=v^{T} \mathbf{f}+v_{0}
$$

where $\mathrm{f}$ is the new feature and $v_{0}$ is an offset value. The calculation result of $y(\mathrm{f})>0$ means that the feature $\mathrm{f}$ belongs to one class and $y(\mathrm{f})<0$ means another class.

\section{The Control Scheme For the Multitasking SIMULATION MANIPULATOR}

\section{A. Simulation Manipulator}

The simulation manipulator we used is the KUKA robot built on the Simulink, Matlab, with seven degrees of freedom, as the Fig. 3 shown. Seven degrees of freedom provides the manipulator more flexibility and allow multi-task design. To construct the manipulator, the models of the arm limb are designed in Simulink, including the definition the model shape and their mechanical properties. All the models of the arm limb are illustrated in Fig.4. Connect them with the joint model provided by the Simulink and set the world coordinates and other parameters, the whole manipulator model is constructed.

As discussed earlier, the dynamic control of the manipulator is not the focus of our paper. Therefore, this manipulator only needs us to provide the joint trajectory in the Simulink model of "signal builder", it will move as desired. According to the requirements of our task, we design a set of motions by directly giving the joint trajectories, which is shown in Fig.5. According to these trajectories, the KUKA manipulator will move to finish a task, as shown in Fig.6

\section{B. Toggle-Confirmation Mechanism}

Because EEG control has the characteristics of few recognition types and low recognition accuracy, there are a lot of limitations when EEG directly control the manipulator,

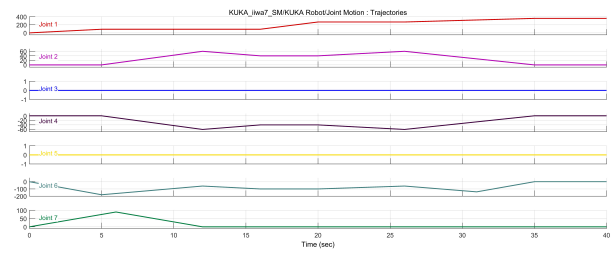

Fig. 5. The trajectories of seven joints in a task

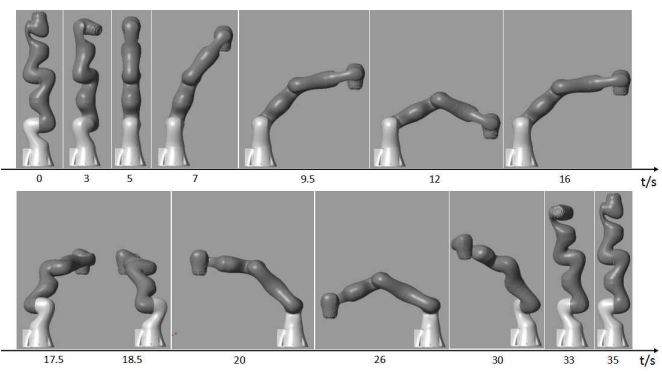

Fig. 6. The movement of the KUKA manipulator in a task

such as few control actions and high error rate. So, in this case, it is difficult for the manipulator to finish a series of actions. In addition, if we increase the EEG recognition types, the recognition accuracy will be reduced, which means that two-category recognition can get the highest accuracy in most cases. However, two-category recognition is not enough for the manipulator control. Therefore, a toggle-confirmation mechanism is proposed here to improve the application of the mind controlled manipulator.

As the Fig.7 shown, it is the interface of the toggle-confirm mechanism, which is controlled by the two classified result of the EEG signal, motor imaginary of left and right-hand movement. First of all, we assume that the motor imagery of left-hand movement is to switch the tasks menu, and the righthand movement is to confirm this task. There is a menu with four main tasks, and each main task corresponds to the three sub-tasks and a cancel option. For example, if we want to select the task $3 \Rightarrow$ subtask2 to let the manipulator turn on the light, we should switch the main tasks menu to the "Task3". Every time we image left-hand movement for about $2 \mathrm{~s}$, this Imagination will be detected and classified as a command sent to the toggle-confirm mechanism, and the tasks menu will be switch one step. The current task we switch to will be lit up to cue the user. When we switch the main task to the "Task3", image right-hand movement to confirm it, then the tasks menu will enter into its subtask. In the same way, we can switch the subtasks menu to the "Subtask2". After confirming this subtask by imaging right-hand movement, the manipulator will turn on the light. Before the task is completed, the manipulator will not receive any control commands and the tasks menu will return to "Task 1" after the task is finished. With this mechanism, two input signals can perform 12 tasks, or even more. 


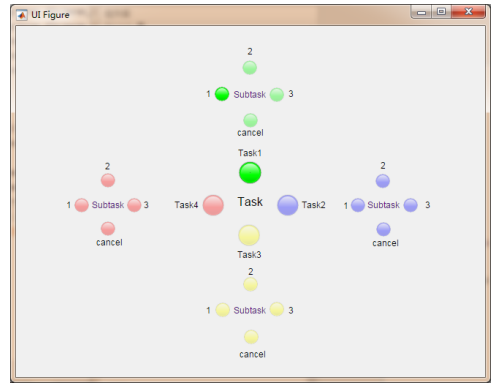

Fig. 7. The interface of the toggle-confirm mechanism

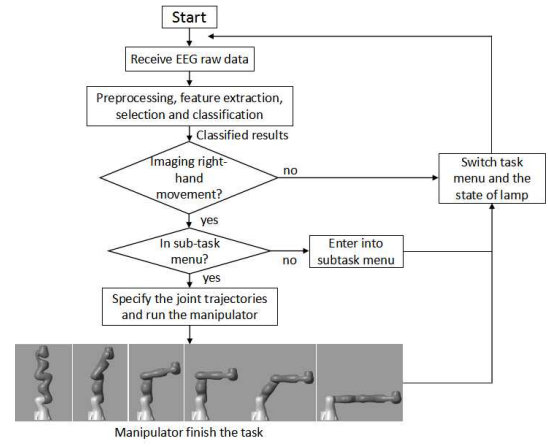

Fig. 8. The flow chart of the control system

\section{The Realization of Toggle - Confirmation Mechanism}

The interface of the toggle-confirm mechanism is designed as an app by the MATLAB plug-in, App Designer, which was launched in MATLAB R2016a as the GUIDE alternative products. As the Fig.7 shown, a task corresponds to a pilot lamp unit. First of all, we drag these pilot lamp unit into the design view, set up their initial state, such as the label, position, color, light-off state etc. After that, the rudiments of this app are already formed. An app is like a C++ class, so we can apply for an object of this class in the MATLAB workspace. Then, to realize the connection between this toggle-confirm mechanism and the control of the KUKA manipulator, we create two public functions, the "Toggle" function and the "Confirm" function. As their names, "Toggle" function is used to switch the tasks menu and the "Confirm function is used to confirm the task. Switching the tasks menu only need to change the on-off state of the pilot lamp but when the task is confirmed, we need to finish two works. Firstly, specify the joint trajectories for the manipulator according to which task we confirmed by calling the MATLAB function of "signalbuilder()". Secondly, run the simulation manipulator by the MATLAB function of "sim()". Then, the manipulator will do the task as we want. The flow chart of the whole control system is shown in Fig.8

\section{EXPERIMENT AND RESULT}

\section{A. Experimental setup of the On-Line BCI}

EEG raw data were collected by Neuroscan device with 27 electrodes channels, which can be seen in Fig.9. The EEG

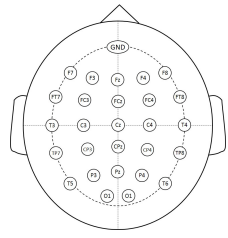

Fig. 9. Scalp map of the 27 channel

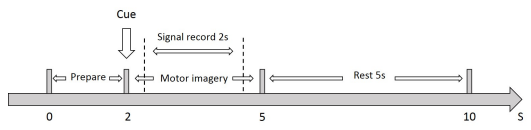

Fig. 10. Time segmentation of one trial

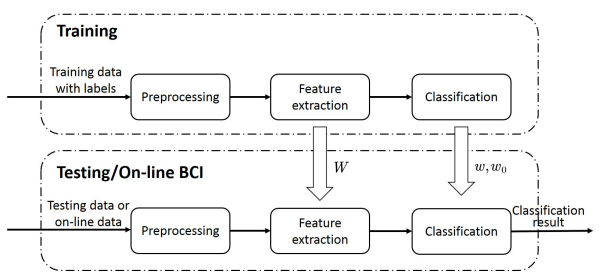

Fig. 11. BCI flow chart

sampling rate was set to $250 \mathrm{~Hz}$ and there is a bandpass filter of 0.5-40 Hz used in the SCAN4.5. As shown in Fig.10, the length of each trial was $10 \mathrm{~s}$, including $2 \mathrm{~s}$ for preparation, $3 \mathrm{~s}$ for motor imagery and $5 \mathrm{~s}$ for resting.

All the procedures of the data processing are shown in Fig.11. Before controlling the manipulator, we must test and verify the accuracy of EEG classification. First of all, we conducted 180 sets of trials according to the above experimental setup, 100 sets for BCI training and the other 80 sets for testing. In BCI training, training data with labels would be preprocessed, feature extracted and classified. Feature extraction is aimed at obtaining the desired spatial filters $W$ and the purpose of classification is to obtain the optimal projection matrix $v$ as well as an offset $v_{0}$. Once these three parameters were defined, models of feature extraction and classification were established, which would be used in next step of testing or on-line EEG data processing.

\section{B. The result of experiment}

There were three male participants for this study, all of whom are from the South China University of Technology. Our procedures were carried out with the adequate understanding and consent of them.

As mentioned earlier, when people image unilateral limb movements, there would be an ERP/ERS phenomenon in their brain. To obtain this phenomenon, We firstly carried out bandpass filtering for the EEG raw data and then processed it with Fourier transform. After that, the phenomenon was presented as shown in Fig.12, where the C3, C4 is the electrode located in the left and right brain respectively. 

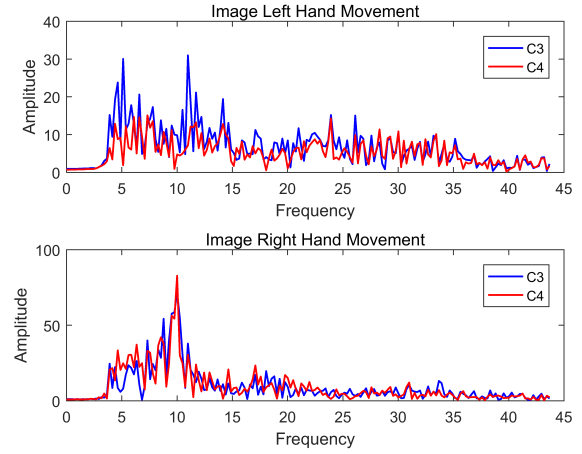

Fig. 12. ERS/ERD phenomenon in the experiment

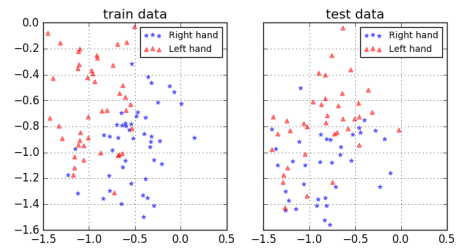

Fig. 13. Classification result

According to the data processing methods mentioned in section II and the experimental setup in the last subsection, we preprocessed, feather extracted, and classified the EEG raw data and got the train and test data accuracy. Fig.13 Show the classification result of one subject. We can see that the train and test data can be well separated, although the test data is relatively poor. The classification accuracy of the three subject is shown in table I. The average test accuracy of them is close to $70 \%$, which is a guarantee for the manipulator control.

After that, the subjects just needed to switch the task menu by imaging left-hand movement and confirm their selection by imaging right-hand movement. And then, the simulation manipulator would do series of actions to fulfill the task, as the Fig.14 shown

TABLE I

CLASSIFICATION ACCURACY

\begin{tabular}{ccc}
\hline Subjects & Train accuracy & Test accuracy \\
\hline S1 & $89 \%$ & $70.00 \%$ \\
S2 & $88 \%$ & $68.75 \%$ \\
S3 & $91 \%$ & $66.25 \%$ \\
Average & $89.33 \%$ & $68.33 \%$ \\
\hline
\end{tabular}

\section{The efficiency of the multi-task manipulator system}

The efficiency of this multi-task manipulator system is an important index to evaluate its application. Firstly, experiments were carried out to test the running time of the EEG data processing program. After testing, the time to acquire the CSP space transformation matrix $W$ for the 100 sets of training data is $12.53 \mathrm{~ms}$ and the time of obtaining the LDA transformation

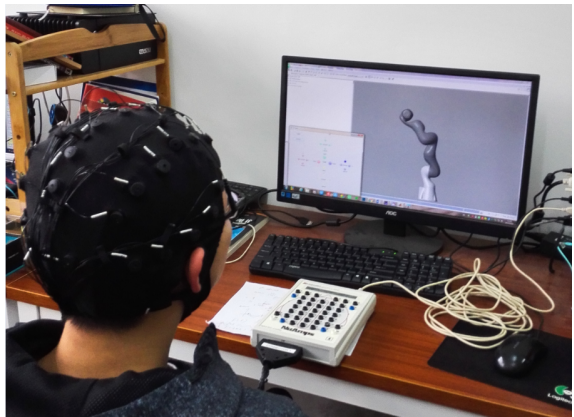

Fig. 14. The scene of manipulator control

matrix $v$ and offset $v 0$ is $1.91 \mathrm{~ms}$. Next, the newly collected EEG raw data were processed. For each trial, there were $64.67 \mathrm{~ms}$ taken for multi-band bandpass filtering and $12.75 \mathrm{~ms}$ taken for CSP feature extraction. Two features were selected by mutual information feature selection and it cost $6.69 \mathrm{~ms}$. Finally, LDA linear classification took $0.0083 \mathrm{~ms}$. It can be seen that after training CSP and LDA model, the processing time of each set of EEG data is less than $100 \mathrm{~ms}$. Since each set of EEG data needs $2 \mathrm{~s}$ for motor imagery, the whole system generates a control instruction about $2.1 \mathrm{~s}$, which can meet the requirement of real-time control because of the low real-time requirement in toggle-confirm mechanism.

In addition, we tested the efficiency of the overall system in task selection. In the experiment, we first assume that each task has a completion time of $10 \mathrm{~s}$, and we do experiments on four representative tasks: task $1 \Rightarrow$ subtask 2 , task $2 \Rightarrow$ subtask2, task $3 \Rightarrow$ subtask 2 , task $4 \Rightarrow$ subtask 2 . The experiment records the time from the beginning to the end of task selection, and the results shown in table II are obtained from participants S1 for ten trails.

TABLE II

TIME SPEND ON TWELVE TASKS SELECTION

\begin{tabular}{ccccc}
\hline Number & $\begin{array}{c}\text { Task1 } \Rightarrow \\
\text { Subtask2 }\end{array}$ & $\begin{array}{c}\text { Task2 } \Rightarrow \\
\text { Subtask2 }\end{array}$ & $\begin{array}{c}\text { Task3 } \Rightarrow \\
\text { Subtask2 }\end{array}$ & $\begin{array}{c}\text { Task4 } \Rightarrow \\
\text { Subtask2 }\end{array}$ \\
\hline 1 & $23.17 \mathrm{~s}$ & $159.09 \mathrm{~s}$ & $69.80 \mathrm{~s}$ & $19.86 \mathrm{~s}$ \\
2 & $70.42 \mathrm{~s}$ & $124.73 \mathrm{~s}$ & $213.31 \mathrm{~s}$ & $170.13 \mathrm{~s}$ \\
3 & $50.55 \mathrm{~s}$ & $10.49 \mathrm{~s}$ & $27.18 \mathrm{~s}$ & $120.67 \mathrm{~s}$ \\
4 & $9.43 \mathrm{~s}$ & $120.70 \mathrm{~s}$ & $18.32 \mathrm{~s}$ & $54.31 \mathrm{~s}$ \\
5 & $88.34 \mathrm{~s}$ & $22.94 \mathrm{~s}$ & $54.61 \mathrm{~s}$ & $134.38 \mathrm{~s}$ \\
6 & $56.68 \mathrm{~s}$ & $26.83 \mathrm{~s}$ & $52.91 \mathrm{~s}$ & $116.59 \mathrm{~s}$ \\
7 & $21.42 \mathrm{~s}$ & $50.28 \mathrm{~s}$ & $227.45 \mathrm{~s}$ & $51.65 \mathrm{~s}$ \\
8 & $78.61 \mathrm{~s}$ & $13.10 \mathrm{~s}$ & $42.20 \mathrm{~s}$ & $146.19 \mathrm{~s}$ \\
9 & $9.33 \mathrm{~s}$ & $126.30 \mathrm{~s}$ & $141.33 \mathrm{~s}$ & $77.20 \mathrm{~s}$ \\
10 & $54.42 \mathrm{~s}$ & $25.07 \mathrm{~s}$ & $17.55 \mathrm{~s}$ & $170.29 \mathrm{~s}$ \\
Average & $46.24 \mathrm{~s}$ & $67.95 \mathrm{~s}$ & $77.47 \mathrm{~s}$ & $106.13 \mathrm{~s}$ \\
\hline
\end{tabular}

The average time of task selection was 74.48s. It means that the multi-task manipulator system is relatively inefficient, on the one hand, because the classification accuracy of EEG is not high enough, on the other hand, there are so many tasks that when in the task switching process, once the error occurs, it will spend extra time. Therefore, we reduce the number of 
tasks from 12 to 4 , we also carry out ten sets of experiments, and get the following results as the table III shown.

TABLE III

TIME SPEND ON FOUR TASKS SELECTION

\begin{tabular}{ccccc}
\hline Number & Task1 & Task2 & Task3 & Task4 \\
\hline 1 & $3.43 \mathrm{~s}$ & $6.71 \mathrm{~s}$ & $54.02 \mathrm{~s}$ & $25.07 \mathrm{~s}$ \\
2 & $33.62 \mathrm{~s}$ & $40.31 \mathrm{~s}$ & $68.45 \mathrm{~s}$ & $14.01 \mathrm{~s}$ \\
3 & $4.11 \mathrm{~s}$ & $6.67 \mathrm{~s}$ & $9.03 \mathrm{~s}$ & $38.73 \mathrm{~s}$ \\
4 & $26.12 \mathrm{~s}$ & $27.79 \mathrm{~s}$ & $8.89 \mathrm{~s}$ & $49.44 \mathrm{~s}$ \\
5 & $3.93 \mathrm{~s}$ & $6.24 \mathrm{~s}$ & $46.59 \mathrm{~s}$ & $12.86 \mathrm{~s}$ \\
6 & $3.36 \mathrm{~s}$ & $51.60 \mathrm{~s}$ & $19.97 \mathrm{~s}$ & $42.34 \mathrm{~s}$ \\
7 & $4.47 \mathrm{~s}$ & $38.73 \mathrm{~s}$ & $80.48 \mathrm{~s}$ & $51.71 \mathrm{~s}$ \\
8 & $73.29 \mathrm{~s}$ & $6.54 \mathrm{~s}$ & $32.17 \mathrm{~s}$ & $38.63 \mathrm{~s}$ \\
9 & $3.77 \mathrm{~s}$ & $5.92 \mathrm{~s}$ & $34.55 \mathrm{~s}$ & $43.07 \mathrm{~s}$ \\
10 & $4.04 \mathrm{~s}$ & $72.47 \mathrm{~s}$ & $17.33 \mathrm{~s}$ & $57.81 \mathrm{~s}$ \\
Average & $16.01 \mathrm{~s}$ & $26.30 \mathrm{~s}$ & $37.15 \mathrm{~s}$ & $37.37 \mathrm{~s}$ \\
\hline
\end{tabular}

The average selection time is about $29.21 \mathrm{~s}$. Obviously, the time spent on task selection has been greatly reduced, basically within the acceptable range.

\section{Discussion AND CONCLUSION}

To address the issue of limited control actions and low accuracy in the EEG controlled manipulator, a multi-task manipulator based on the combination of the motor imagery EEG algorithm and the toggle-confirm mechanism is developed. In the design of BCI, a two-category classification algorithm is used, including multi-band bandpass filtering, CSP for feature extraction, mutual information for feature selection and LDA for classification. As a result, the EEG signals was classified into two categories, left- and right-hand motor imageries, and the test accuracy is around $70 \%$, which ensure that we can control the manipulator with low error rate. As the core of the multi-task manipulator, the toggle-confirm mechanism is facilitated by the classification results of the motor imagery EEG. With EEG translated to commands of the toggle-confirm operation, two types of control signals can be used to switch and select to different tasks, and subsequently, the manipulator will do the corresponding tasks. In the efficiency test, we found that the multi-task manipulator system with toggle-confirm mechanism has a low real-time requirement, and the time spent in task selection is acceptable when the number of tasks is not too many. So, there is no doubt that it has two important advantages:

1) Because current technology can only distinguish a few types of EEG signals, the manipulator is unable to obtain enough control signals to accomplish complex tasks. To address this problem, toggle-confirm mechanism can be used to select more tasks only by two type of EEG signals.

2) The improvement of the recognition type will lead to the reduction of the EEG recognition accuracy. Therefore, the multitasking manipulator with direct EEG control will face the problem of high error rate, which makes the manipulator can not complete a series of actions. In practical applications, the error of the manipulator will likely cause a lot of trouble. But the movement of the manipulator, which is controlled with the toggle-confirm mechanism, is predetermined, so it will not occur action errors in theory.

In this paper, the multitasking manipulator we propose is a manipulator indirectly controlled through the toggle-confirm mechanism. Inevitably it suffers from the shortcomings of inflexibility, lack freedom, and long response time. Thus, the direct control of EEG signal is still the focus of the BCI research. But the control with toggle-confirm mechanism presents a new idea for our application exactly. This will improve the application prospects of EEG controlled manipulator.

\section{REFERENCES}

[1] J. R. Wolpaw, N. Birbaumer, W. J. Heetderks, D. J. McFarland, P. H. Peckham, G. Schalk, E. Donchin, L. A. Quatrano, C. J. Robinson, T. M. Vaughan et al., "Brain-computer interface technology: a review of the first international meeting," IEEE transactions on rehabilitation engineering, vol. 8, no. 2, pp. 164-173, 2000.

[2] N. Kosmyna, F. Tarpin-Bernard, N. Bonnefond, and B. Rivet, "Feasibility of bci control in a realistic smart home environment," Frontiers in Human Neuroscience, vol. 10, p. 416, 2016.

[3] B. M. Young, Z. Nigogosyan, V. A. Nair, L. M. Walton, J. Song, M. E. Tyler, D. F. Edwards, K. Caldera, J. A. Sattin, and J. C. Williams, "Case report: post-stroke interventional bci rehabilitation in an individual with preexisting sensorineural disability," Frontiers in Neuroengineering, vol. 7, p. 18, 2014.

[4] R. Folgieri and R. Zampolini, "Bci promises in emotional involvement in music and games," Computers in Entertainment, vol. 11, no. 4, pp. $1-10,2015$.

[5] G. R. Mller-Putz, R. Scherer, C. Brauneis, and G. Pfurtscheller, "Steadystate visual evoked potential (ssvep)-based communication: impact of harmonic frequency components," Journal of Neural Engineering, vol. 2, no. 4, pp. 123-130, 2006.

[6] B. Rebsamen, E. Burdet, C. Guan, H. Zhang, C. L. Teo, Q. Zeng, C. Laugier, and M. H. A. Jr, "Controlling a wheelchair indoors using thought," Intelligent Systems IEEE, vol. 22, no. 2, pp. 18-24, 2007.

[7] M. Balconi and G. Mazza, "Brain oscillations and bis/bas (behavioral inhibition/activation system) effects on processing masked emotional cues. : Ers/erd and coherence measures of alpha band," International Journal of Psychophysiology Official Journal of the International Organization of Psychophysiology, vol. 74, no. 2, pp. 158-65, 2009.

[8] L. A. Miner, D. J. Mcfarland, and J. R. Wolpaw, "Answering questions with an electroencephalogram-based brain-computer interface." Archives of Physical Medicine \& Rehabilitation, vol. 79, no. 9, pp. 1029-33, 1998.

[9] N. Birbaumer, T. Elbert, A. G. Canavan, and B. Rockstroh, "Slow potentials of the cerebral cortex and behavior." Physiological Reviews, vol. 70, no. 1, p. 1, 1990.

[10] X. Li, X. Zhao, and T. Tan, "A behavior-based architecture for the control of an intelligent powered wheelchair," in IEEE International Workshop on Robot and Human Interactive Communication, 2000. Ro-Man 2000. Proceedings, 2000, pp. 80-83.

[11] G. R. Mller-Putz and G. Pfurtscheller, "Control of an electrical prosthesis with an ssvep-based bci," IEEE transactions on bio-medical engineering, vol. 55, no. 1, pp. 361-4, 2008.

[12] T. Li, J. Hong, J. Zhang, and F. Guo, "Brainmachine interface control of a manipulator using small-world neural network and shared control strategy," Journal of Neuroscience Methods, vol. 224, no. 6, pp. 26-38, 2014.

[13] S. A. Al-Shaban, M. S. Al-Faysale, A. Qusai, and H. Al-Neami, "Non-linear principal component analysis neural network for blind source separation of eeg signals," Research Journal of Applied Sciences Engineering \& Technology, vol. 2, no. 2, pp. 180-190, 2010.

[14] X. Li, S. S. Ge, Y. Pan, K.-S. Hong, Z. Zhang, and X. Hu, "Feature extraction based on common spatial analysis for time domain parameters," in Ubiquitous Robots and Ambient Intelligence (URAI), 2011 8th International Conference on. IEEE, 2011, pp. 377-382. 